\title{
Relocalización de las actividades terciarias y cambios en la centralidad en la Región Metropolitana de Buenos Aires ${ }^{1}$
}

\author{
Lorena Vecslir ${ }^{2}$ y Pablo Ciccolella ${ }^{3}$
}

\begin{abstract}
RESUMEN
Entre las transformaciones territoriales más recientes resultan cada vez más significativos los cambios en las lógicas locacionales, configuración física y programas funcionales de los nuevos espacios del terciario. El proceso de crecimiento y difusión territorial de estas actividades (centros de negocios, hotelería, grandes superficies del ocio y el consumo) en la región urbana de Buenos Aires durante las dos últimas décadas se relaciona con las diferentes modalidades de reestructuración de las centralidades y subcentralidades metropolitanas, verificándose tendencias tanto hacia la continuidad de dinámicas, morfologías y localizaciones tradicionales, como a la consolidación de una red de distritos de localización del terciario, compatible con los nuevos patrones metropolitanos mundiales de configuración más dispersa y funcionamiento en red.
\end{abstract}

Palabras clave: Reestructuración territorial, Región Metropolitana de Buenos Aires, actividades terciarias, centralidades metropolitanas.

\begin{abstract}
Among the most recent territorial transformations, changes in the logic of localization, in the physical configuration of space, as well as in the functional programs of the new "tertiary" spaces are increasingly significant. The growth process and the territorial spread of activities such as business center services, lodging, large retail and leisure establishments, in the urban region of Buenos Aires during the last two decades, relates to different restructuring modalities of metropolitan centralities and subcentralities. It seems easy to verify relevant trends such as the continuity of traditional dynamics, morphologies and locations, as well as the consolidation of a district network of service activities location, compatible with the sparser configuration and network operation of new global metropolitan patterns.
\end{abstract}

Key words: Territorial restructuring, metropolitan region of Buenos Aires, service activities, metropolitan centralities.

1 Artículo recibido el 19 de octubre de 2010, aceptado el 18 de marzo de 2011 y corregido el 29 de marzo de 2011.

2 Instituto de Geografía, Universidad de Buenos Aires (Argentina). E-mail: lorena.vecslir@gmail.com

3 Departamento de Geografía, Universidad de Buenos Aires (Argentina). E-mail: pablociccolella@arnet. com.ar 
Las transformaciones económicas y sociales de las últimas décadas han contribuido a generar cambios territoriales, que, entre otros fenómenos, se expresan en tendencias hacia una nueva estructura urbana y metropolitana. A su vez, estos cambios en el territorio replantean la distribución geográfica, configuración física y programas funcionales de los nuevos espacios del terciario.

Los avances tecnológicos, las transformaciones organizacionales, las nuevas formas de producción, trabajo, consumo y opciones residenciales desde los años ochenta, están definiendo tendencias hacia un nuevo modelo de ciudad, hacia el replanteo de la propia naturaleza de lo urbano, que se expresa particularmente en un nuevo tipo de suburbio, articulado por una serie de nuevos objetos urbanos, o artefactos de la globalización: centros comerciales, urbanizaciones privadas, ampliación de accesos y redes de autopistas metropolitanas, parques temáticos, centros de ocio, parques empresariales, industriales, logísticos, entre otros.

Si bien la problemática de trasfondo de este trabajo está vinculada a la discusión ciudad compacta-ciudad dispersa, en particular, en esta instancia el centro estará en el análisis del proceso de difusión, crecimiento y localización de los nuevos espacios del terciario (centros de negocios, hotelería, grandes superficies de ocio y consumo) en la Región Metropolitana de Buenos Aires (RMBA) ${ }^{4}$, desde los años noventa hasta la actualidad (Figura $\mathrm{N}^{\mathrm{o}} 1$ ).

\footnotetext{
4 Se entiende por RMBA a las siguientes jurisdicciones: a) Ciudad Autónoma de Buenos Aires (CABA), con un área de $200 \mathrm{~km}^{2}$ y una población actual de alrededor de 3.100.000 habitantes; b) Gran Buenos Aires (GBA), conformada por 24 partidos (departamentos o municipios) que forman la primera y segunda corona de la aglomeración; y c) el resto de la RMBA o tercera corona, integrada por 16 partidos, con un área aproximada de $15.800 \mathrm{~km}^{2}$ y una población actual de 1.600 .000 habitantes. En conjunto, la RMBA comprende un área de $19.680 \mathrm{~km}^{2}$ y una población actual aproximada de 13.700.000 habitantes. La expresión AMBA (Área Metropolitana de Buenos Aires), se utilizará para denominar al conjunto CABA+GBA, y la expresión RMBA (Región Metropolitana de Buenos Aires), para el conjunto total de la aglomeración.
}

Los cambios en los factores de localización geográfica y en los nuevos contenidos del terciario avanzado, estarían contribuyendo a la formación de subcentros metropolitanos, que presentan algunas características de complementariedad con la centralidad tradicional (microcentro extendido y antiguos centros departamentales de la región). Asimismo, las transformaciones en estudio estarían redefiniendo las tendencias en el uso del espacio metropolitano, que se acerca más al funcionamiento de una red de geometría variable que al del tradicional movimiento centro-periferia, retroalimentando un proceso de suburbanización ya no exclusivamente basado en la residencia, sino que incorpora progresivamente nuevos espacios de actividades y de trabajo, vinculados al comando empresarial, al ocio, al consumo, al comercio y a los servicios a la producción y a las personas.

A partir del análisis del proceso de crecimiento y localización territorial de las actividades terciarias, este trabajo se propone interpretar las diferentes modalidades de reestructuración de las centralidades y subcentralidades metropolitanas; y en términos más amplios, aportar nuevos argumentos metodológicos y elementos conceptuales a la discusión sobre la estructura, morfología y naturaleza de las principales mutaciones territoriales de las dos últimas décadas en la RMBA.

El artículo se divide en tres apartados. En el primero se realiza una síntesis de las principales tendencias en la estructuración de los territorios metropolitanos en el contexto del capitalismo global y de las nuevas tecnológicas de comunicación, con especial mención al apogeo de la ciudad corporativa y los distritos de comando en las grandes metrópolis latinoamericanas, y en particular, en la RMBA. En el segundo, se describe el proceso de crecimiento, modernización y localización territorial de las actividades terciarias en esta región, a partir de la confección de una base de datos del terciario corporativo (en edificios de nueva planta o reequipados con tecnologías inteligentes), y de otras actividades de servicio como las grandes superficies comerciales y de ocio. Finalmente, se realiza una interpretación de las dinámicas locativas relevadas y se plantean, a manera de hipótesis, algunas tendencias recurrentes en la configuración de la centralidad metropolitana y redefinición territorial del poder económico. 
Figura $\mathrm{N}^{0} 1$

Región Metropolitana de Buenos Aires (RMBA)



Fuente: Elaborado por Marianela Figueroa para el Programa de Estudios sobre Desarrollo Territorial y Estudios Metropolitanos (PDTEM), Instituto de Geografía, Universidad de Buenos Aires.

Desde el punto de vista metodológico, cabe aclarar que se ha trabajado a partir de diversas fuentes de información (reportes inmobiliarios, suplementos de periódicos especializados y trabajo de terreno), sobre el segmento de oficinas clase $A^{5}$, considerando

5 Dentro del mercado de oficinas, las clases A y A+ (o AAA) hacen referencia a edificios para alquiler, generalmente bien localizados, con un alto nivel de diseño, amenities, sistemas tecnológicos y ma- que se trata del producto inmobiliario que, en forma individual o dentro de parque de negocios o centros corporativos, mejor expre-

nagement. La mayor parte de estos edificios son de nueva construcción, aunque las operaciones de reciclado pueden posicionar antiguos edificios dentro de esta categoría. En algunos mercados existe lo que se denomina edificios inteligentes, que se diferencian del resto por su alto grado de tecnología, valor agregado en el management e imagen corporativa (Reporte Inmobiliario, 2003). 
sa la expansión del sector terciario (tanto en metros cuadrados construidos como en nuevas localizaciones y nivel de innovación en la gestión empresarial), y que, junto a otros sectores (grandes emprendimientos residenciales, comerciales y de equipamiento privado), está contribuyendo a la reestructuración de la RMBA.

\section{Las tendencias actuales en la producción del territorio metropolitano}

Las dinámicas territoriales están cada vez más vinculadas a los ciclos económicos y particularmente a los cambios en las lógicas locacionales que se desprenden de las transformaciones tecnológicas, productivas y organizacionales del sistema capitalista. Podría decirse inclusive, que debido a la ascendente importancia que adquieren los territorios metropolitanos en los mecanismos de acumulación, en razón de su complejidad, escala y densidad productiva, la etapa actual de desarrollo de dicho sistema económico podría caracterizarse como capitalismo metropolitano.

La nueva armazón de asentamientos y las nuevas jerarquías, así como la indiferenciación técnica y cultural del espacio rural frente al urbano y el consumo creciente del primero por parte del segundo en los bordes metropolitanos, hace que se vaya estructurando un territorio reticular que tiende a reemplazar a los territorios-zona (Veltz, 1996). La tensión emerge claramente: territorios-zona que resisten, apegados a estructuras productivas, sociales y políticas tradicionales, con fuerte dependencia del aparato estatal como soporte de la armazón socioterritorial; frente a territorios-red que emergen como resultado de la formación de un medio técnico-científicoinformacional (Santos, 1996) o de un modo de producción informacional (Castells, 1995). En términos de Carlos de Mattos (2008), este fenómeno desemboca en la producción de un nuevo patrón general de urbanización que denomina "lo urbano generalizado". Así, las jerarquías se vuelven multiescalares, trazando en el espacio mallas sumamente complejas y superpuestas, donde las relaciones tienden a hacerse cada vez más horizontales, saltándose los peldaños intermedios de la jerarquía christalleriana (Ascher, 1995).
En términos espaciales, se ha ido pasado de un territorio urbano continuo que avanzaba en forma de mancha de aceite, con una morfología y bordes bastante bien definidos, hacia un crecimiento metropolitano en red, conformando una verdadera ciudad-región, de bordes difusos, policéntrica, constituyendo en algunos casos, megalópolis o archipiélagos urbanos. En otras palabras, se pasa de territorios estructurados fundamentalmente en base a la articulación horizontal y contigua de los lugares o regiones, a un territorio estructurado tridimensional y verticalmente por medio de redes y en forma de red (Ciccolella, 1999).

Luego de un proceso dialéctico de desestructuración-reestructuración a partir del nuevo régimen de acumulación postfordista, la estructura y morfología metropolitana tiende a ser regenerada. Cada formación territorial metropolitana regenera a su vez la relación entre estas etapas del ciclo económico, con mayor énfasis en alguno de ellos, pero con una tendencia general a su creciente fusión en el marco del continuo industria-servicios, en un complejo económico-territorial que Castells (1995) caracteriza como informacional. Por su parte, Pierre Veltz (1994) y Saskia Sassen (1994) coinciden en señalar a la expansión del mercado de los servicios especializados o avanzados como responsables del nuevo auge y de las nuevas dinámicas metropolitanas, ya que es en este tipo de entramados territoriales, donde aquellos se han desarrollado vertiginosamente en los últimos años, constituyéndose en sí mismos en factor de atracción de las grandes empresas y por lo tanto de reforzamiento del proceso de remetropolización.

Las ciudades latinoamericanas no resultan ajenas a estos procesos. En estas metrópolis, las grandes corporaciones presentan patrones de comportamiento territorial específicos, donde ellas mismas son factor protagónico de la construcción del territorio y, por lo tanto su territorialidad es, en buena medida autogenerada (Lobato Corrêa, 1994). En el caso de la RMBA, nunca como antes, el poder económico deconstruyó y construyó (o reconstruyó) nuevas estructuras territoriales con la libertad que lo hizo en los años noventa.

Asimismo, las constantes innovaciones en materia de sistematización y difusión de la 
información, potencian la importancia estratégica de los procesos de toma de decisiones, y, paradójicamente se tornan el principal factor del proceso de reconcentración territorial del poder económico. Según Cordeiro (1993) "a pesar de la telemática, los momentos de toma de decisiones, las discusiones sobre investigaciones científicas, el intercambio de asuntos confidenciales de negocios se realizan cara a cara" (Khon Cordeiro, 1993: 324).

En ese proceso el área central de las grandes metrópolis, o "ciudad corporativa" (Ciccolella, 1999: 8) cumple, entre otras funciones, la de alojar las sedes centrales de las principales entidades bancarias locales y globales. Estas desempeñan un papel destacado en el proceso de circulación del capital, contribuyendo a incrementar el carácter de nodo de gestión y del poder económico de las ciudades donde se aglomeran, y en particular, de sus espacios centrales (Lobato Corrêa, 1993).

La problemática de la centralidad implica un análisis a doble escala (Alessandri Carlos, 2001). La escala de la propia región metropolitana como centro o como nodo de un sistema de ciudades y territorios a los que se conecta, o que a veces comanda; y la escala del núcleo central de la aglomeración donde se concentran las funciones de comando (sedes empresariales, sedes financieras, servicios avanzados, hotelería internacional, centros políticos, centros culturales, entre otros). En síntesis, hablar hoy de centralidad implica, por un lado, la delimitación de unas zonas de concentración de equipamientos, funciones y actividades vinculadas al comando de la economía regional, nacional y del capitalismo global o "distritos de comando" (Ciccolella, 1999: 17). Por otro lado, la definición de la centralidad, implica considerar la red completa de centros y subcentros que constituyen un único espacio de control transaccional y corporativo.

Así, la configuración polinuclear de la centralidad, a la manera de Santiago de Chile o de San Pablo, con grandes operaciones urbanísticas que han tendido a desplazar la centralidad clásica en términos físicos, o bien, una estructura polinuclear tenue en forma de corredor corporativo que no afecta la hegemonía y expansión de la centralidad tradicional, como sucede en Buenos Aires, constituyen en cualquier caso un único espa- cio de comando que se inserta en la red de ciudades globales o nodos de control de la economía global.

Un fenómeno que en la RMBA se retroalimenta especialmente en los años 90 cuando paralelamente a la renovación, expansión y mejora de la red de autopistas, se diversifican los procesos de suburbanización residencial, se modifican las condiciones locacionales del sector comercial y de servicios, y las industrias refuerzan tendencias ya existentes asociadas con las autopistas (Blanco, 1999). Se trata del paso del aglomerado a la red, según el cual tienden a definirse nuevas centralidades o subcentralidades metropolitanas de estructura compleja, donde el crecimiento ya no se produce en continuidad con el tejido urbano existente sino que se implanta a lo largo de los grandes ejes de circulación.

\section{La localización territorial de las actividades terciarias en la RMBA}

Dentro de este marco, actualizando y completando las bases de datos de algunos trabajos anteriores (Ciccolella, 2000; Ciccolella y Lucioni, 2005; Ciccolella y Baer 2008; Vecslir y Ciccolella, 2009), se ha reconstruido el proceso de crecimiento, modernización y localización territorial de las actividades terciarias en la RMBA, indagando en torno al comportamiento de este sector, mediante observaciones de campo y datos del mercado inmobiliario (superficie construida, precio de alquileres, tasa de vacancia), como el segmento funcional más explicativo del poder del capital económico financiero en la redefinición de la estructura urbana territorial.

Tal como puede observarse en el Cuadro $N^{\circ} 1$, durante los años noventa, la mayor parte del terciario corporativo (en nuevos desarrollos o edificios aggiornados a las tecnologías recientes) se localiza en el área central de la RMBA. Las zonas clave de concentración son: el microcentro, la plaza San Martín o Retiro, el macrocentro y Catalinas Norte. Fuera del área central tradicional, el 15\% de los desarrollos se realizan en Puerto Madero, y otro porcentaje similar entre el resto del macrocentro y el eje norte de la CABA (desde Recoleta hasta Saavedra). 
En el corredor norte de la RMBA (desde la Av. Gral Paz -autopista de circunvalación de la Ciudad Autónoma de Buenos Aires- hasta el Partido de Tigre) se registra un $13 \%$ del total de la superficie de oficinas disponible en ese periodo, marcando el inicio de un proceso de cambio en el patrón tradicional de localización de la actividad terciaria corporativa que, unido al proceso de descentralización de los espacios del ocio y el consumo, tiende a generar nuevas subcentralidades nodales o lineares a escala metropolitana, como se verá más adelante.

Pasada la crisis de 2001 y la devaluación, muchas empresas (tecnológicas, exportadoras, servicios financieros, representaciones de bancos, entre otras) se vieron atraídas por la baja de los precios de las oficinas clase A y decidieron mudarse a esas unidades, incluso a aquellas localizadas fuera del área central tradicional. Así, según el reporte de mercado de la consultora Castro Cranwell \& Weiss (2002-2006), el primer semestre de 2003 mostró cierta reactivación en las operaciones correspondientes a oficinas $\mathrm{A}$ y $\mathrm{A}+$ que otorgaron dinamismo al mercado, gracias a los usuarios de grandes superficies que, aprovechando la baja de los precios de alquiler, se trasladan a edificios de mayor categoría.

Entre el 2004 y el 2005 se afirma esta tendencia y la vacancia comienza a descender. Si bien el único gran emprendimiento en la CABA es el Bouchard Plaza (edificio La Nación, en plaza Roma), aparecen nuevos proyectos y reciclado de edificios medianos (entre $3.000 \mathrm{~m}^{2}$ y $5.000 \mathrm{~m}^{2}$ ), destacando las zonas del Macrocentro, Barracas, Palermo, y los partidos de Vicente López y San Isidro. Según la misma fuente citada, en los años siguientes la demanda continúa insatisfecha y hace que los valores de alquiler de las oficinas clase A sigan subiendo. Las rentas se

\section{Cuadro $\mathrm{N}^{\mathrm{o}} 1$}

Total de superficie construida de edificios inteligentes* entre 1990 y 2001 por zonas de la RMBA

\begin{tabular}{|c|c|c|c|c|}
\hline \multicolumn{2}{|r|}{ Zona } & $\begin{array}{l}\text { Superficie } \\
\qquad\left(\mathrm{m}^{2}\right)\end{array}$ & $\begin{array}{c}\text { Superficie en relación a } \\
\text { Ciudad de Buenos Aires (\%) }\end{array}$ & $\begin{array}{c}\text { Superficie en } \\
\text { relación a RMBA }(\%)\end{array}$ \\
\hline \multirow{3}{*}{$\begin{array}{l}\text { Área } \\
\text { central } \\
\text { tradicional } \\
\text { de la CBA }\end{array}$} & Microcentro & 528.759 & 25,78 & 22,40 \\
\hline & Catalinas Norte & 216.523 & 10,56 & 9,17 \\
\hline & Plaza Roma & 65.368 & 3,19 & 2,77 \\
\hline \multirow{8}{*}{$\begin{array}{l}\text { Otras áreas } \\
\text { de la CBA }\end{array}$} & Plaza San Martín & 343.062 & 16,73 & 14,53 \\
\hline & Macrocentro & 215.507 & 10,51 & 9,13 \\
\hline & Subtotal & 1.369 .219 & 66,76 & 57,99 \\
\hline & Puerto Madero & 309.653 & 15,10 & 13,12 \\
\hline & Resto Macrocentro & 185.665 & 9,05 & 7,86 \\
\hline & Zona Sur CBA & 61.763 & 3,01 & 2,62 \\
\hline & "Eje norte" CBA & 129.799 & 6,33 & 5,50 \\
\hline & Subtotal & 681.752 & 33,24 & 28,88 \\
\hline \multicolumn{2}{|c|}{ Subtotal Ciudad de Buenos Aires } & 2.050 .971 & 100,00 & 86,87 \\
\hline \multicolumn{2}{|c|}{ "Corredor norte" de la CBA } & 309.977 & - & 13,13 \\
\hline \multicolumn{2}{|c|}{ Total RMBA 1990-2001 } & 2.360 .948 & - & 100,00 \\
\hline
\end{tabular}

* Se incluyen edificios de calidad, construidos entre 1930 y 1970, reciclados y reequipados con sistemas de control inteligente dentro del período considerado en este cuadro.

Fuente: Elaborado en base a Ciccolella y Lucioni (2005). 
vuelven atractivas para los emprendedores e inversores y comienzan a surgir nuevos proyectos de grandes superficies (entre $8.000 \mathrm{~m}^{2}$ y $30.000 \mathrm{~m}^{2}$ ) en el área este de Puerto Madero (Buenos Aires Plaza, Dique 3), eje norte de la CABA (Working Ocampo en Barrio Parque), partido de San Isidro (Centro Uruguay Norte I), km 50 de la Panamericana (Concord Pilar I y II, Edificio Bureau II), entre otros.

Pero mientras que en el 2007 e inicios del 2008 el porcentaje de oficinas disponibles era casi nulo, a partir del 2009 la vacancia del segmento $\mathrm{A}$ y $\mathrm{A}+$ asciende al $10 \%$ y $7 \%$, respectivamente, y la creciente disponibilidad de espacios provoca un freno a la subida de precios que se venía dando en forma casi constante desde 2005, entrando en una fase de "amesetamiento" y estabilidad de valores. En este periodo se inaugura la torre corporativa de Repsol-YPF y el Madero Center (Dique 3 y 4 de Puerto Madero); se recicla el edificio Cruz de Malta en la zona sur y el del Banco de Galicia en el microcentro de la ciudad; y en la zona norte de la región destacan los desarrollos del Olivos Center, y Panamerican Bureau en el partido de Vicente López; Polo Uno, Blue Building, Skyglass-Ayres Vila, y la primera etapa del Parque Austral (edificios Insignia) en el partido de Pilar.

Así, desde el 2002 la zona norte del GBA se destaca por ser la de mayor crecimiento en edificios de oficinas, incorporando más de

Cuadro $\mathrm{N}^{\circ} 2$

Total de superficie construida de edificios clase A y A+* entre 2002 y 2010 por zonas de la RMBA

\begin{tabular}{|c|c|c|c|c|}
\hline & Zona & $\begin{array}{c}\text { Superficie } \\
\left(\mathrm{m}^{2}\right)\end{array}$ & $\begin{array}{c}\text { Superficie en relación } \\
\text { a Ciudad de Buenos } \\
\text { Aires }(\%)\end{array}$ & $\begin{array}{c}\text { Superficie en } \\
\text { relación a RMBA } \\
(\%)\end{array}$ \\
\hline Área central & Microcentro & 72.981 & 11,75 & 6,08 \\
\hline tradicional & Catalinas Norte & - & 0,00 & 0,00 \\
\hline & Plaza Roma & 43.100 & 6,94 & 3,59 \\
\hline & Plaza San Martín & 2.000 & 0,32 & 50,00 \\
\hline & Macrocentro & 61.087 & 9,84 & 5,09 \\
\hline & Subtotal & 179.168 & 33,61 & 14,92 \\
\hline Otras áreas & Puerto Madero & 247.032 & 39,78 & 13,8 \\
\hline de la CBA & Zona Sur CBA & 86.050 & 13,86 & 2,6 \\
\hline & "Eje norte" CBA & 108.823 & 17,52 & 5,4 \\
\hline & Subtotal & 441.905 & 71,15 & 29,5 \\
\hline Subtotal CBA & & 621.073 & 100,00 & 51,71 \\
\hline GBA & "Corredor norte" GBA & 533.106 & - & 44,39 \\
\hline & Zona oeste GBA & 42.900 & - & 3,57 \\
\hline & Zona sur GBA & 4.000 & - & 0,33 \\
\hline Subtotal GB & & 580.006 & - & 48,29 \\
\hline Total RMBA & $2-2010$ & 1.201 .079 & - & 100,00 \\
\hline
\end{tabular}

* Se incluyen edificios de calidad, construidos entre 1930 y 1970, reciclados y reequipados con sistemas de control inteligente dentro del período considerado en este cuadro.

Fuente: Elaboración propia en base a Suplementos semanales de Inmuebles Comerciales del Diario La Nación, período 2002-2010. 
$500.000 \mathrm{~m}^{2}$ al mercado y representando casi un $45 \%$ del crecimiento total en ese periodo $\left(1.201 .079 \mathrm{~m}^{2}\right)$ y un $23 \%$ del total generado desde los años 90 hasta la actualidad (Cuadro $\mathrm{N}^{\circ} 2$ ). Cabe destacar en este sentido, operaciones de gran envergadura (entre $10.000 \mathrm{~m}^{2}$ y $50.000 \mathrm{~m}^{2}$ ) como los complejos Urbana, Optima Business Park, Work Center y Libertador 350, así como los proyectos del Torre Regatta Oficinas Panamericana 1050, Edificio AWWA, Proa y Nodus Business District, todos ellos en el municipio de Vicente López.

El mapa de la distribución territorial de edificios de oficinas A y A+ entre 1990-2010 en la RMBA (Figura $N^{\circ} 2$ ) enseña que a la zona tradicional de demanda corporativa (Catalinas, Plaza Roma, Plaza San Martín, microcentro), ahora extendida al macrocentro y especialmente a la zona de Puerto Madero, hay que sumar dentro de la ciudad de Buenos Aires, el corredor de los barrios de Palermo, Belgrano, Núñez y más recientemente Saave- dra, así como algunos sectores embrionarios de desarrollo en la zona sur con el reciclado de edificios emblemáticos, como es el caso del barrio de Barracas y el recientemente creado distrito tecnológico de Parque de los Patricios.

En la zona norte de la región, las áreas que presentan mayor concentración de edificios corporativos se estructuran alrededor de tres ejes paralelos a la costa del Río de la Plata:

- El corredor de la Panamericana (con algunos nodos significativos entorno a los centros comerciales y de ocio UnicenterNorcenter y a la altura de Pilar);

- El corredor de la Av. del Libertador (especialmente en la zona cercana al río en $\mathrm{Vi}$ cente López), que se completa con calles transversales como Paraná en Martínez y Dardo Rocha en San Isidro;

- Y las Av. Maipú, Santa Fe y Centenario.

Figura $N^{\circ} 2$

Distribución territorial de edificios A y A+ entre 1990-2010 en la RMBA

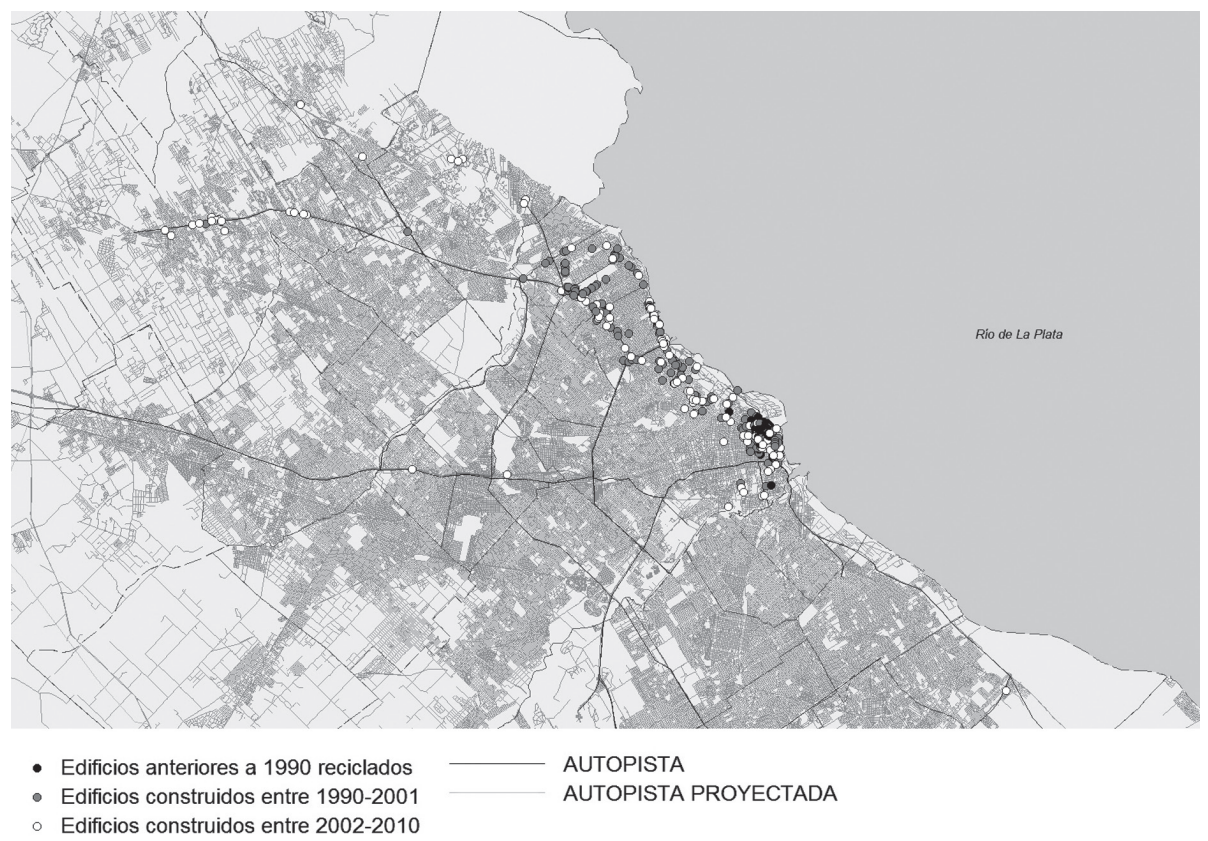

Fuente: Elaboración en base a relevamientos propios. Cartografía digital: Marianela Figueroa. 
Este patrón de localización permite afirmar que se estaría ante un proceso incipiente de descentralización, extendida hacia el suburbio norte, a lo largo de las principales vías de comunicación, con algunos nodos de mayor densidad donde estas actividades conviven con otras ligadas al ocio-consumo, los servicios hoteleros, y los grandes equipamientos de salud y educativos. Entre otros motivos, esta predilección creciente y estable por la zona norte se relaciona, con:

- La alta accesibilidad (desde la autopista Panamericana o viarios arteriales);

- La diferencialidad en el mercado de suelo (menores valores de compra o alquiler y mayor disponibilidad de metros cuadrados, a igual nivel de calidad de construcción y servicios que en el área central tradicional);

- El control o seguridad, disponibilidad de plazas de estacionamiento y modernidad edilicia que proveen los nuevos complejos corporativos;

- La revalorización del concepto de "trabajar en el verde" (búsqueda de mayor tranquilidad, entornos parquizados, vistas al río);

- La proximidad a la residencia de gran parte de los directivos de las empresas, radicados en los countries y barrios cerrados existentes en la zona;

- Y el progresivo cambio cualitativo del suburbio, en cuanto a complejización funcional e incorporación de equipamientos de calidad.

A diferencia de las oficinas, el patrón de localización de las grandes cadenas internacionales de hipermercados (Carrefour, Jumbo) y nacionales (Norte, Coto y Tia S. A.) tiende en cambio a vincularse desde un inicio (años 80) con la zona norte del CUB. A partir de mediados de los años 90 se produce una importante fase de expansión con la llegada de nuevos competidores extranjeros (cadena estadounidense Walmart), se genera una explosión de nuevas aperturas o hipermercadización (Ciccolella, 2000), presentando actualmente un esquema de distribución territorial mucho más homogéneo que el resto de actividades terciarias (Figura $\mathrm{N}^{\circ} 3$ ).
Los shopping centers, en cambio, tienden a coincidir con las zonas de mayor concentración de oficinas, destacando entre las últimas inauguraciones (DOT Baires Shopping, Paseo Pilar, Maschwitz Mall, Buenos Aires Mall, Pilar Point, entre otros), la polarización de centros en relación al Acceso Norte en el cruce con la Gral. Paz, y en los Ramales Escobar y Pilar.

Estos nuevos nodos de consumo suelen completarse con centros de ocio (o multicines) y home centers (superficies especializadas en artículos para el hogar), configurando parques comerciales. En relación a los complejos multipantalla, existe un cambio a partir de la llegada a la RMBA, en 1997, de las cuatro cadenas de capital extranjero Hoyts General Cinemas, Village Cinemas, Cinemark y Cines Showcase, compañías que han inaugurado 23 multicines (con un total de 228 salas), presentes en los shoppings o conformando ellos mismos centros de ocio. Las principales cadenas de home centers, Easy (de capitales germano-chilenos perteneciente al Grupo Cencosud) y Sodimac (de capitales chilenos perteneciente al Grupo Falabella), se instalan en la región en los años 1993 y 2008 respectivamente y, a diferencia de los otros formatos, el mayor número de implantaciones se localiza en la zona norte.

\section{La reestructuración de la centralidad. Diferentes modalidades de un mismo proceso}

La elaboración de cartografía específica y el análisis del proceso de crecimiento y difusión territorial de las actividades terciarias en la RMBA durante las dos últimas décadas permiten una interpretación de las dinámicas en curso, observándose la consolidación de algunas tendencias en el proceso de relocalización y redefinición territorial del poder económico. Así, como primera aproximación, se diferencian seis modalidades de reestructuración de las centralidades y subcentralidades metropolitanas. 
Figura $\mathrm{N}^{\circ} 3$

Distribución territorial de los centros del ocio y el consumo en la RMBA

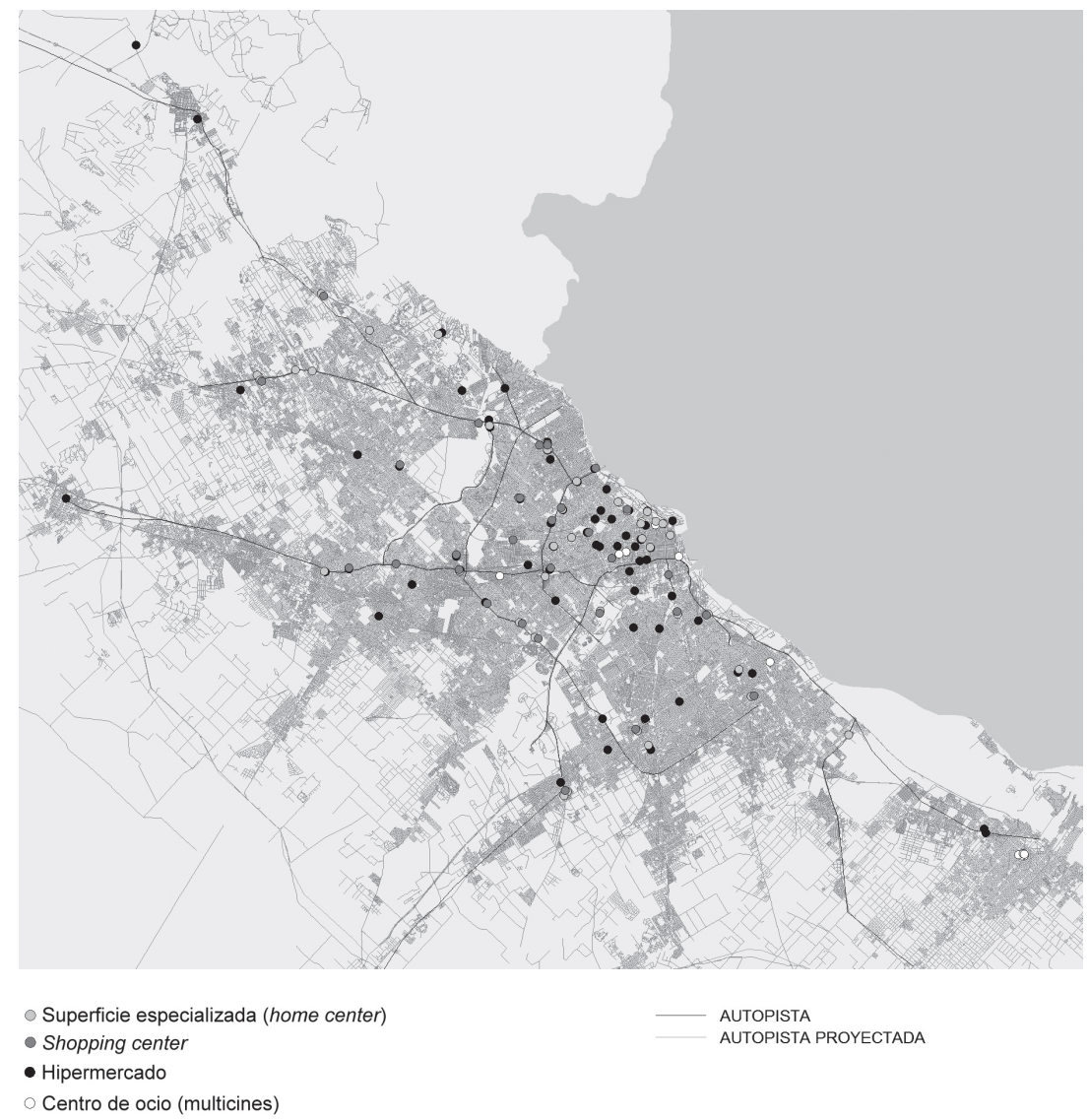

Fuente: Elaboración en base a relevamientos propios. Cartografía digital: Marianela Figueroa.

\section{a) Modernización, verticalización y densificación del área central tradicional}

Tal como puede observarse en los cuadros precedentes y Figuras $\mathrm{N}^{\circ} 2$ a $\mathrm{N}^{\circ} 4$, esta área aglutina el mayor porcentaje de superficie de edificios corporativos, existiendo algunas zonas más densas que otras, como el caso del Microcentro, plaza San Martín, plaza Roma, y Catalinas Norte, las cuales se encuentran más verticalizadas en altura (Ciccolella y Lucioni, 2005), y donde aún se registran procesos importantes de modernización de edificios corporativos, así como las últimas oportunidades de desarrollo de nuevos em- prendimientos $^{6}$. En este sector continúan concentrándose la mayor parte de las actividades de gobierno central y local, el grueso de la actividad hotelera internacional y de las sedes bancarias.

\footnotetext{
6 En este sentido, cabe mencionar la reciente venta por subasta de dos importantes terrenos en el distrito empresarial céntrico de Catalinas (restando un tercero) que pertenecían a la Ciudad de Buenos Aires y donde se podrán construir alrededor de $150.000 \mathrm{~m}^{2}$ de oficinas, hoteles y comercios.
} 


\section{b) Consolidación del área central "expandida"}

La formación de un área central que se derrama hacia el norte y particularmente hacia el este y sur, fortalecida por la operación urbanística de Puerto Madero, genera por su contigüidad espacial un efecto de estiramiento del centro tradicional, más que un centro de negocios alternativo (Ciccolella y Lucioni, 2005). Estas áreas de expansión se encuentran en un claro proceso de consolidación, albergando además un importante número de equipamientos y servicios ${ }^{7}$ (Figura $\mathrm{N}^{\circ} 4$ ).

\section{c) Mixtura residencial-laboral en barrios tradicionales}

En los barrios del eje norte de la ciudad existe un proceso creciente de mixtura de nuevos edificios corporativos con el tejido residencial y la oferta comercial existente (avenidas principales o shopping centers como Alto Palermo, Solar de la Abadía, DOT Baires Shopping, entre otros). Mientras que en Palermo Hollywood se podría hablar de una difusión del office boutique, tipología de oficinas de mediana altura y superficie, a veces con amenities, pensada para estudios de jóvenes profesionales, productoras de cine y televisión ${ }^{8}$, agencias de publicidad o comunicación, entre otros; en Belgrano, Núñez, y Saavedra surgen grandes torres corporativas, atraídas por la accesibilidad del corredor de la Av. del Libertador, la extensión del subte D,

7 Sirve como ejemplo, la concentración de servicios informáticos y consultoras en los docks reciclados del sur de Puerto Madero -Daycom, 3COM, Tekbridge, entre otros- $y$ en la zona contigua de San Telmo -Oracle, IBM, DTK Computer Inc., Exxo-.

8 El apogeo de la localización de la denominada industria de la producción audiovisual, en los barrios porteños de Palermo, Colegiales, Chacarita, Villa Ortúzar y Paternal, se desarrolló durante buena parte de los años 2000. Actualmente, el sector atraviesa una cierta crisis de crecimiento y maduración. En este contexto algunas productoras han comenzado a optar por trasladarse a áreas del Conurbano Norte, fuera de la CABA, a efectos de abaratar costos fijos. Ante esta realidad el Poder Ejecutivo porteño envió a la Legislatura, en julio de 2010, un proyecto de creación de un Polo Audiovisual, con la intención de retener y potenciar esa industria, con incentivos similares al del Distrito Tecnológico Buenos Aires, cuyas características se detallan en la nota siguiente. sobre el potente corredor comercial de la Av. Cabildo y/o la rápida conexión hacia la Panamericana $y$, en general, la zona norte.

\section{d) Reestructuración de antiguas áreas industriales (distrito tecnológico), y reciclado de edificios emblemáticos en la zona sur}

La propuesta e incentivo público a la formación de un distrito tecnológico en los barrios porteños de parque de los Patricios y Nueva Pompeya ${ }^{9}$, junto al proyecto de rehabilitación de antiguos edificios industriales (Palacio Lezama -compañía alimentaria Canale-, empresa yerbatera Cruz de Malta, antigua fábrica de Alpargatas, entre otros) y algunos emprendimientos importantes en el barrio de Barracas, han aportado una significativa cantidad de metros cuadrados, tanto en el segmento de edificios de oficinas como de procesos productivos, a la zona sur de la ciudad, manteniendo en cierta medida su antigua identidad industrial y ofreciendo tipologías alternativas al centro tradicional, en lo relativo a la amplitud y altura de las plantas libres y su menor coste manteniendo una localización central.

9 Mediante la Ley 2.972 de diciembre de 2008, la Legislatura de la Ciudad Autónoma de Buenos Aires creó el Distrito Tecnológico Buenos Aires (DTBA), con epicentro en los barrios porteños de Parque Patricios y Nueva Pompeya. El emprendimiento, algo polémico, recoge parcialmente la experiencia del proyecto "22@" de Barcelona, y se propone impulsar un proceso de puesta en valor y transformación urbana, creando una herramienta de apoyo a la competitividad de las empresas TIC, a través de incentivos tales como exención del pago de ingresos brutos y ABL por diez años, del pago de los impuestos de sellos y la posibilidad de diferir impuestos para ser invertidos en obras. Además, contarán con incentivos fiscales en materia de derechos de alineación y construcciones por el mismo lapso. Por otro lado, los empleados de las empresas que se instalen en el distrito y que se muden al barrio también estarán exentos de pagar ABL por diez años y tendrán líneas de créditos disponibles a tasas preferenciales. Las entidades académicas también accederán a beneficios impositivos. Cabe señalar también, a propósito de este emprendimiento que el tercer Banco Oficial más importante del país, el Banco Ciudad, ha decidido trasladar su sede central a dicho Distrito, en parque Patricios. 
Figura $\mathrm{N}^{\circ} 4$

Distribución territorial de edificios A y A+ entre 1990-2010 en la RMBA.

Detalle del área central de la CABA

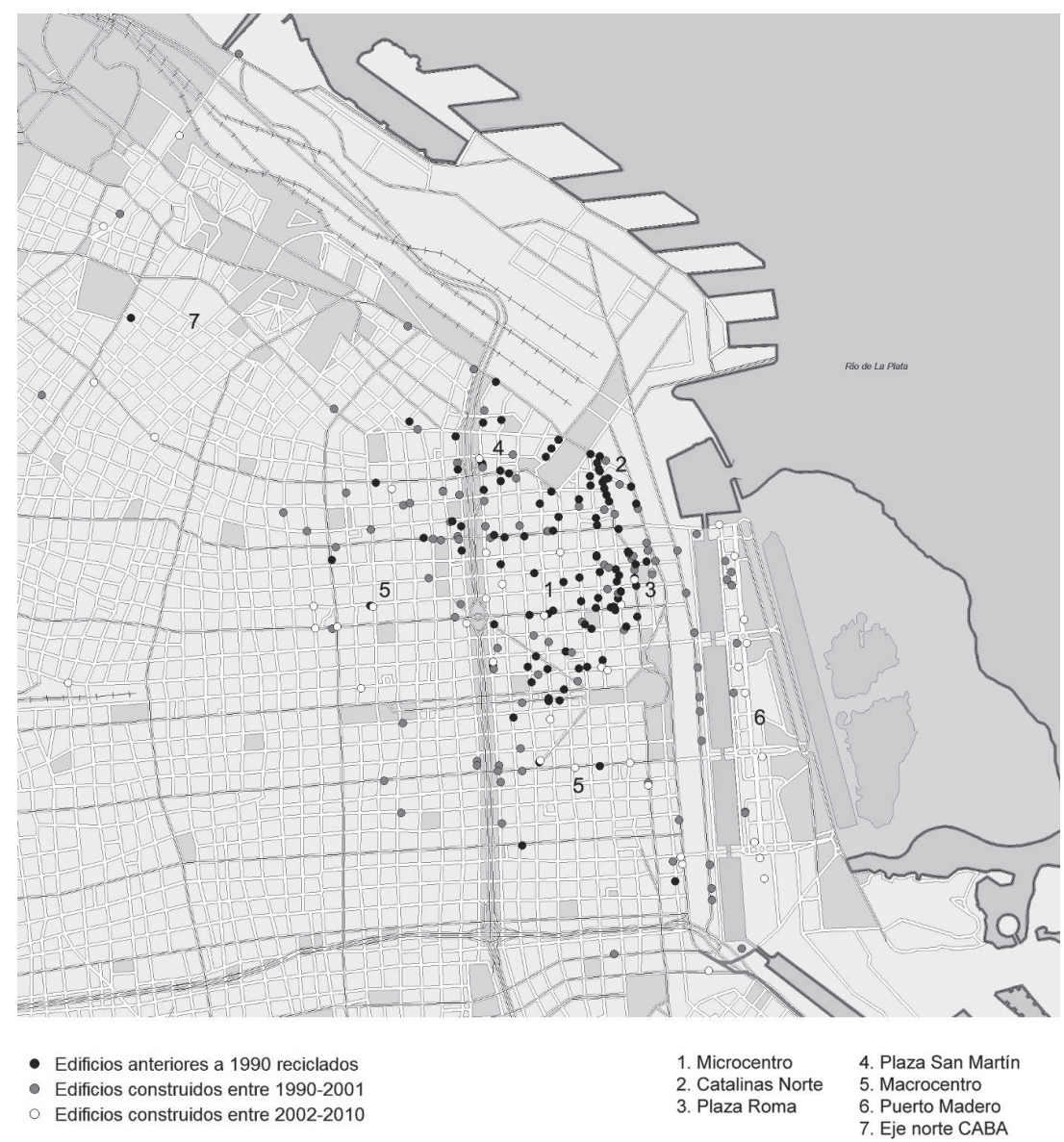

Fuente: Elaboración en base a relevamientos propios. Cartografía digital: Marianela Figueroa.

e) Consolidación y densificación de distritos de comando en Vicente López y San Isidro

La mayor cantidad de oferta de actividades terciarias desarrollada en los últimos años se emplaza en los partidos de Vicente López y San Isidro, especialmente a lo largo de la Av. del Libertador y de la ruta Panamericana, prolongando el eje metropolitano norte mas allá de la General Paz. Este corredor concentra las sedes de grandes empresas nacionales y multinacionales ${ }^{10}$, junto a otras pequeñas y medianas, despachos profesionales y servicios.

\footnotetext{
10 Entre las más importantes en facturación tienen sede en zona norte: Peugeot, Acindar, Carrefour, Cencosud, Nestlé, Alto Paraná, IBM, Bayer, Makro, Avon, Capsa, Roche, Carrier, Prosegur, The Value, Brand Company, Agroservicios Pampeanos; así como otras
} 
En las inmediaciones de Panamericana, en torno a los centros comerciales Norcenter y Unicenter, aparece un foco de oferta de oficinas clase $A$, mientras que en Vicente López se está formando un importante polo corporativo en la zona de la Av. del Libertador al río, priorizando las visuales a la ribera. Los anteriores sectores se complementan con otros corredores de menor densidad e importancia formados por la Av. Maipú-Santa Fe-Centenario, y algunas calles transversales como Paraná en Martínez y Dardo Rocha en los alrededores del Hipódromo de San Isidro y el Jockey Club de Buenos Aires (Figura No 5).

\section{f) "Concentración discontinua" en} municipios emergentes de los corredores Norte y Oeste de la RMBA

De manera discontinua, el proceso se extiende a lo largo de la autopista Panamericana Ramal Pilar, destacando los nodos conformados a la altura del km 42 y 49,5 de dicha arteria. El crecimiento residencial de partido de Pilar, generado por la proliferación de urbanizaciones (clubes de campo y barrios cerrados), se ve complementado actualmente por una importante oferta ocio-comercial (Paseo Pilar, Las Palmas del Pilar, Torres del Sol, Villages, entre otros) y educativa (colegios y universidades privadas) que, sumada al desarrollo del parque industrial y la rápida accesibilidad de las autopistas, configura un polo de gran atracción para las empresas que buscan instalar sus oficinas administrativas y comerciales. Los primeros complejos (Bureau Pilar, Concord Pilar y Office Park) se inauguran a finales de los 90, y las segundas etapas se conciben a mediados de la década siguiente. Recientemente han surgido nuevos emprendimientos en el acceso al núcleo urbano (Pilar Point) y en el km 42,5 y 53,5 de la autopista (Blue Building, Skyglass-Ayres Vila y Polo Uno). Más al interior, en relación a la ruta 8 , cabe mencionar el desarrollo de los edificios insignia de la primera etapa del

empresas de primer nivel nacional e internacional como: Citibank, BMW, Nike Argentina, Adidas, Johnson \& Johnson, Laboratorios Sidus, Merck Sharp \& Dohme, Havanna, Bonafide, Disney, Compaq y Motorola (Ramos Brokers Inmobiliarios, 2007). parque científico y tecnológico de la Universidad Austral.

Pero además de estos nodos y corredores corporativos que se afianzan con los proyectos en curso, entre los km 42 y 50 del Acceso Norte, debe tenerse en cuenta la emergencia de nuevos complejos corporativos como aquellos localizados en el partido de Tigre (Riviera Park, Puerta Norte II, Santa María Park) que surgen sobre la base de un gran crecimiento demográfico impulsado por la operación Nordelta. O el incipiente desarroIlo terciario a la altura del km 26 del Acceso Oeste, en proximidad del cruce con el camino del Buen Ayre, basado en la formación del polo automotriz Parque Leloir donde se han instalado grandes superficies comerciales e importantes empresas (Toyota, Nissan, Pirelli, Yamaha, Citroën, Ford, Peugeot, Easy Home Center, Mapfre), y donde comienzan a surgir nuevas oficinas A+ (Bureau Leloir), acompañando desde la autopista, actividades del ocio y el consumo en forma de parques y "paseos" comerciales, así como complejos de edificios inteligentes, en recintos parquizados con proceso de suburbanización residencial entre los partidos de Ituzaingó y Luján.

Por lo tanto, se verifica la consolidación de una red de subcentros en la zona norte de la región (Ciccolella y Lucioni, 2005). Una concentración discontinua de la actividad terciaria, que reúne en emplazamientos de localización estratégica y acceso directo desde la autopista, actividades del ocio y el consumo en forma de parques y paseos comerciales, así como complejos de edificios inteligentes, en recintos parquizados con perímetro controlado, ofreciendo la posibilidad de "comprar y trabajar en el verde".

\section{Consideraciones finales}

Al cabo de dos décadas de transformaciones morfológicas y estructurales en la RMBA y particularmente en lo referente a la evolución de los nuevos espacios del terciario, es posible afirmar que se observan procesos heterogéneos y complejos tanto en las áreas de localización tradicional de dichas actividades como en aquellas que precisamente han ido surgiendo desde principios de los años noventa. 


\section{Figura $N^{\circ} 5$}

Distribución territorial de edificios A y A+ entre 1990-2010 en la RMBA.

Detalle de los partidos de Vicente López y San Isidro

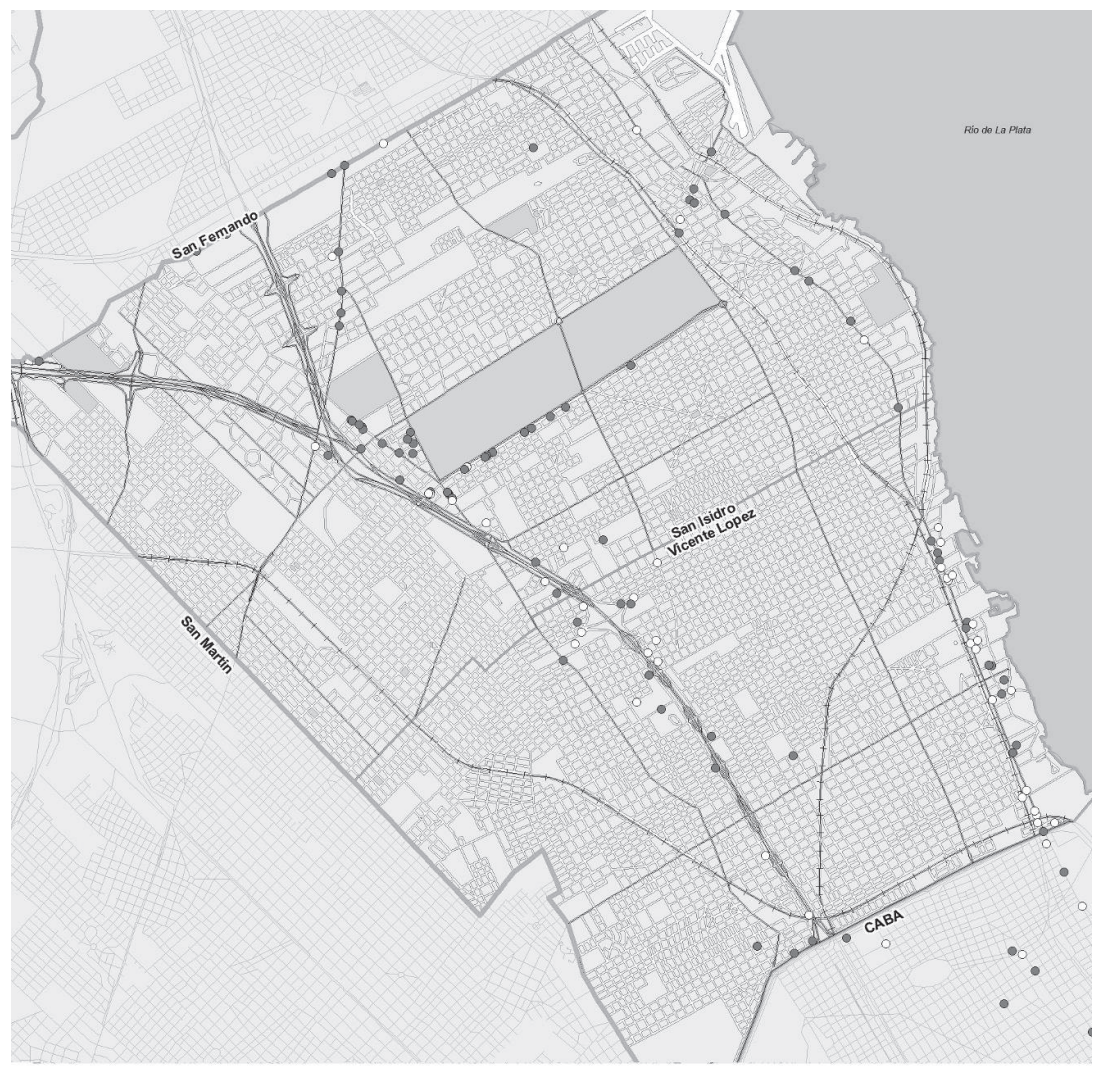

- Edificios anteriores a 1990 reciclados

- Edificios construidos entre 1990-2001

- Edificios construidos entre 2002-2010

Fuente: Elaboración en base a relevamientos propios. Cartografía digital: Marianela Figueroa.

Las tensiones y dinámicas que se verifican en el conjunto de la región permiten afirmar, que continúan observándose tendencias tanto hacia la continuidad de viejas dinámicas, morfologías y localizaciones, vinculadas a cierta tradición monocéntrica de Buenos Aires, como a la aparición y consolidación de una red de distritos de localización del terciario más compatible con los nuevos conceptos y tendencias metropolitanas mundiales, hacia la ciudad dispersa, ciudad región, ciudad-red, etc.
Sin embargo, si hacia principios de los años dos mil podíamos afirmar que la incipiente red de distritos de comando de la RMBA no tendía a competir, sino a complementarse con la centralidad tradicional expandida, hacia fines de los dos mil se abre el interrogante sobre la existencia de un freno o "techo" a la oferta de espacio y equipamientos para el terciario avanzado en el área central tradicional y una arrolladora dinámica de producción de suelo y proyectos para alojar y/o relocalizar esas funciones, especialmente en 
el Eje Norte de la RMBA, aunque ya se perfilan también otras alternativas, como el Eje Oeste.

La precariedad y escasez de instrumentos y voluntad política de regular y ordenar estas tendencias desde las autoridades públicas de la Ciudad y la Provincia de Buenos Aires resultan insuficientes y con dificultades adaptativas para asimilar este tipo de procesos. La excepción son las políticas públicas de beneficios fiscales y respaldo crediticio para la atracción de empresas en el Parque Austral, Distrito tecnológico de Parque de los Patricios, Distrito Audiovisual, etc. Sin embargo, aun en estos casos, no se reconocen criterios en relación al paisaje urbano, obras de equipamiento o espacio público.

La problemática de la gobernanza surge entonces con fuerza en estos procesos, y pone en tela de juicio tanto los alcances de los poderes locales como la necesidad de formas de articulación y actuación coordinada a nivel metropolitano de la ciudad concreta. Se advierte la necesidad de un plan de escala territorial, acordado entre las administraciones municipales, a fin de redistribuir y compensar las cargas y beneficios, y para un desarrollo de los futuros centros y subcentros coordinado con los servicios y las redes de infraestructuras existentes y previstas, racionalizando las nuevas implantaciones y el consumo de suelo.

Aunque no lo hemos desarrollado en este trabajo, también se observan tendencias espontáneas, es decir, vía mercado, a la revitalización de las áreas centrales de las cabeceras municipales de varios partidos bonaerenses, especialmente en la zona sur. Por lo tanto, sería muy pertinente promover paralelamente, políticas de recuperación, mantenimiento y potenciación de dichas subcentralidades tradicionales.

Pero el desafío pendiente, quizá de mayor envergadura, está centrado en la necesidad de rearticulación de la relación entre nodos y "corredores" existentes y potenciales, dentro de un esquema territorial en red, ya que los procesos espontáneos y de control del capital sobre el territorio ya han asumido esta realidad, mientras que el urbanismo y la planificación territorial pública apenas tienen conciencia de ello.

\section{Referencias bibliográficas}

ALESSANDRI CARLOS, A. Espaco-Tempo na metrópoli. São Paulo: Contexto, 2001.

ASCHER, F. Métapolis. Ou l'avenir des viIles. Paris: Editions Odile Jacob, 1995.

BLANCO, J. Transporte y espacio urbano en Buenos Aires: Reestructuración de la red de autopistas metropolitanas y cambios en la organización espacial. En: Buenos Aires, 1er Encuentro Internacional Humboldt, 1999.

CASTELLS, M. La ciudad informacional. Tecnologías de información, reestructuración económica y el proceso urbano-regional. Madrid: Alianza Editorial, 1999.

CICCOLELLA, P. Globalización y dualización en la Región Metropolitana de Buenos Aires. Grandes inversiones y reestructuración socioterritorial en los años 90. EURE, 1999, vol. 25, $\mathrm{N}^{\circ} 76$, p. 5-27.

CiCCOLELLA, P. Distribución global y territorio. Modernización y concentración comercial en Argentina en los años noventa. Economía, Sociedad y Territorio, 2000, vol. II, $\mathrm{N}^{\circ} 7$, p. 459-496.

CICCOLELLA, P. y BAER, L. Buenos Aires tras la crisis: ¿Hacia una metrópolis más integradora o más excluyente? Ciudad y Territorio, Estudios Territoriales, 2008, Vol. XL, No 157, p. 641-660.

CICCOLELLA, P. y LUCIONI, N. La ciudad corporativa. Nueva arquitectura empresarial, redefinición de la centralidad y surgimiento de una red de distritos de comando en la Región Metropolitana de Buenos Aires. En: DE MATTOS, C. et al. (eds.). Gobernanza, competitividad y redes: la gestión en las ciudades del siglo XXI. Santiago: Instituto de Estudios Urbanos y territoriales, Pontificia Universidad Católica de Chile, Colección RIDEAL y EURELibros, 2005, p. 185-209.

DE MATTOS, C. La tercera revolución urbana en América latina ¿Hacia lo urbano generalizado? En: Querétaro, X Seminario Internacional RII, 2008. 
KHON CORDEIRO, H. A 'cidade mundial' de São Paulo e o complexo corporativo do seu centro metropolitano. In: SANTOS, M. et al. (edit.). Fim de século e globalização. São Paulo: Hucitec-Anpur, 1993, p. 318-331.

LOBATO CORRÊA, R. Dinâmica do espaço financeiro brasileiro 1961-1985. In: CAPUANO SCARLATO, F. et al. (org.). Globalização e espaço latino-americano. São Paulo: Hucitec-Anpur, 1993, p. 163-168.

LOBATO CORRÊA, R. Territorialidade e corporação: un exemplo. In: SANTOS, M. et al. (org.). Território. Globalização e Fragmentação. São Paulo: Hucitec-Anpur, 1994, p. 251-256.

RAMOS BROKERS INMOBILIARIOS, L. J. Oficinas: una tendencia que viene para quedarse. Informe del mercado inmobiliario, octubre de 2007, p. 2-6.

REPORTE INMOBILIARIO. Clasificación de oficinas. Buenos Aires: Reporte Inmobi- liario, 2003. Disponible em Internet: http:// www.reporteinmobiliario.com/nuke/article88clasificacion-de-oficinas.html

SANTOS, M. A natureza do espaço. Ténica e tempo. Razão e emoção. São Paulo: HUCITEC, 1996.

SASSEN, S. El complejo urbano en una economía mundial. Revista RICS, 1994, № 139, p. 55-78.

VELTZ, P. Des territoites pour apprendre et innover. Paris: Editions de l'aube, 1994.

VELTZ, P. Mondialisation, Villes et Territoires. L'Économie d'Archipel. Paris: Presses Universitaires de France, 1996.

VECSLIR, L. y CICCOLELLA, P. Nuevos territorios del ocio y el comercio en la región metropolitana de Buenos Aires (1990-2008). Revista Proyección, 2009, vol. III, N 7. Disponible en Internet: http://cifot.com.ar/proyeccion/archivos_sistema/presentaciones/39.pdf 